\title{
Tuberculosis ganglionar cervical simulando una enfermedad de Kikuchi: Reporte de un caso y revisión de la literatura
}

\author{
Cervical lymph node tuberculosis simulating Kikuchi's disease: \\ A case report and review of the literature
}

\author{
Lisbeth Valoyes $\mathbf{G}^{1}$, Iván García $\mathbf{D}^{1}$, Amadeo Muntané $\mathbf{S}^{1}$, Paloma Mora $\mathbf{M}^{1}$, Lucía Aja $\mathbf{R}^{1}$.
}

\begin{abstract}
RESUMEN
La linfadenitis cervical tuberculosa es la manifestación más común de tuberculosis en cabeza y cuello. La enfermedad de Kikuchi es una enfermedad rara que consiste en una linfadenitis necrotizante histocitiaria. La presentación clínica en ambas entidades suele consistir en una o más masas cervicales dolorosas, que pueden ser duras o fluctuantes. Los hallazgos por imagen no permiten distinguir de manera absoluta la linfadenitis tuberculosa de la enfermedad de Kikuchi. Por lo tanto es imprescindible para interpretar los hallazgos por imagen no sólo conocer el origen demográfico del paciente, sino su estado inmunológico con respecto a la tuberculosis y el estudio histopatológico y microbiológico de las adenopatías.
\end{abstract}

Palabras clave: Enfermedad de Kikuchi, nódulo linfático, linfadenopatía cervical, TC.

\begin{abstract}
Tuberculous cervical lymphadenitis is the most common manifestation of tuberculosis in the head and neck. Kikuchi's disease is a rare disease that is histocitiaria necrotizing lymphadenitis. The clinical presentation in both entities is usually a painful neck masses or more, which can be hard or fluctuating. The imaging findings do not distinguish in absolute tuberculous lymphadenitis Kikuchi's disease. Therefore it is essential to interpret the imaging findings not only know the origin of the patient population, but their immune status with regard to Tuberculosis and histopathological and microbiological lymphadenopathy.
\end{abstract}

Key words: Kikuchi disease, lymph node, cervical lymphadenopathy, CT.

Médicos de Neurorradiología. Departamento de Neurorradiología, Hospital Universitario de Bellvitge. Feixa Llarga s/n 08907 L'Hospitalet de Llobregat, Barcelona-España. 


\section{INTRODUCCIÓN}

La linfadenitis tuberculosa (LT) cervical y la enfermedad de Kikuchi-Fujimoto (EK) son dos patologías que forman parte del diagnóstico diferencial de las linfadenitis necrotizantes en los estudios de diagnóstico por imagen. A continuación presentamos el caso de un paciente joven de origen asiático que presentó una tumoración cervical submaxilar derecha dolorosa. A pesar de que la linfadenitis cervical tuberculosa es la manifestación más común de la tuberculosis (TBC) en cabezay cuello, el diagnóstico inicial por tomografía computarizada (TC) y por tomografía por emisión de positrones (PET) se inclinó hacia EK. Este diagnóstico se realizó considerando el lugar de procedencia del sujeto, la unilateralidad y las características de las adenopatías que mostraban la presencia de múltiples focos necróticos. Además el paciente no tenía antecedentes de tuberculosis (TBC) pulmonar. La biopsia no mostró bacilos ácido-alcohol resistentes (BAAR), y el diagnóstico efectuado histopatológicamente fue de linfadenitis necrotizante. Sin embargo las pruebas complementarias posteriores de quantiferon y la PCR (reacción en cadena de la polimerasa) para tuberculosis fueron positivas igual que el PPD (derivado proteico purificado), confirmándose el diagnóstico de linfadenitis tuberculosa cervical.

Es de nuestro interés, a propósito del caso planteado, realizar una aproximación diagnóstica de las características imagenológicas, principalmente en TC, de la linfadenitis tuberculosa y enfermedad de Kikuchi, aportando también las imágenes del PET y realizar una revisión de la literatura.

\section{PRESENTACIÓN DEL CASO}

Paciente masculino de 25 años, de origen chino, con antecedentes de orquidectomía derecha por una vasculitis granulomatosa necrotizante primaria testicular. No era portador del VIH ni tenía otra causa de inmunodeficiencia. Seis meses después presentó una tumoración laterocervical y submaxilar derecha dolorosa. Se realizó TC de cuello que puso de relieve un grupo de adenopatías cervicales derechas de aspecto heterogéneo con focos hipodensos de aspecto necrosado (Figura 1). Posteriormente se realizó PAAF guiada por ultrasonido cuyo resultado no mostró células malignas. A pesar de ello se realizó un PET que mostró un conglomerado adenopático yugular medio e inferior derecho hipermetabólico, que por sus características era necesario descartar malignidad (Figura 2).

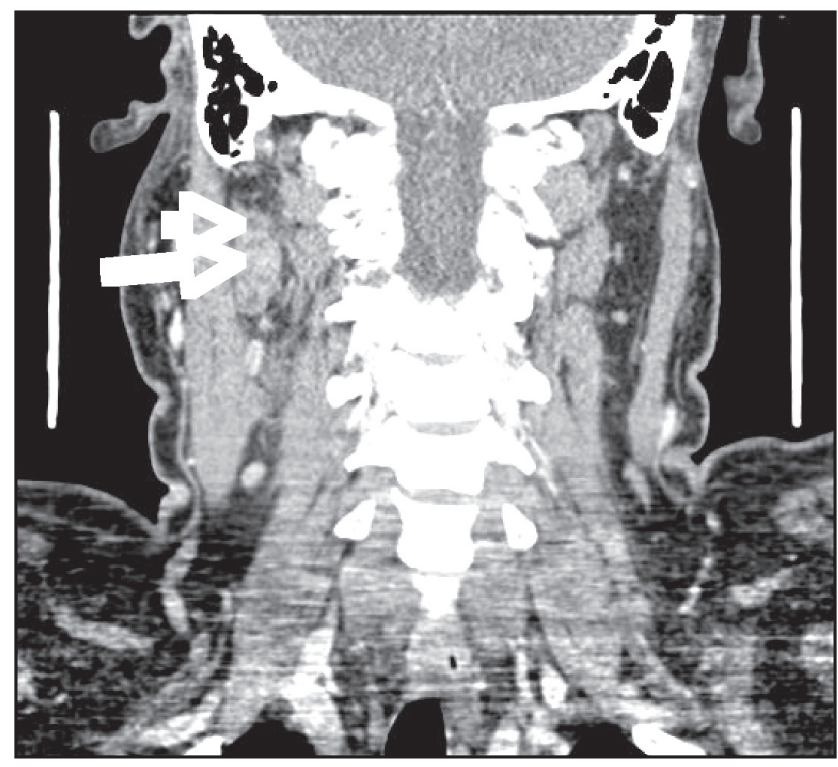

Figura 1. TC de cuello con contraste. Corte coronal. Adenopatía (flecha blanca larga) que presenta pequeños focos de necrosis (flecha blanca corta). 


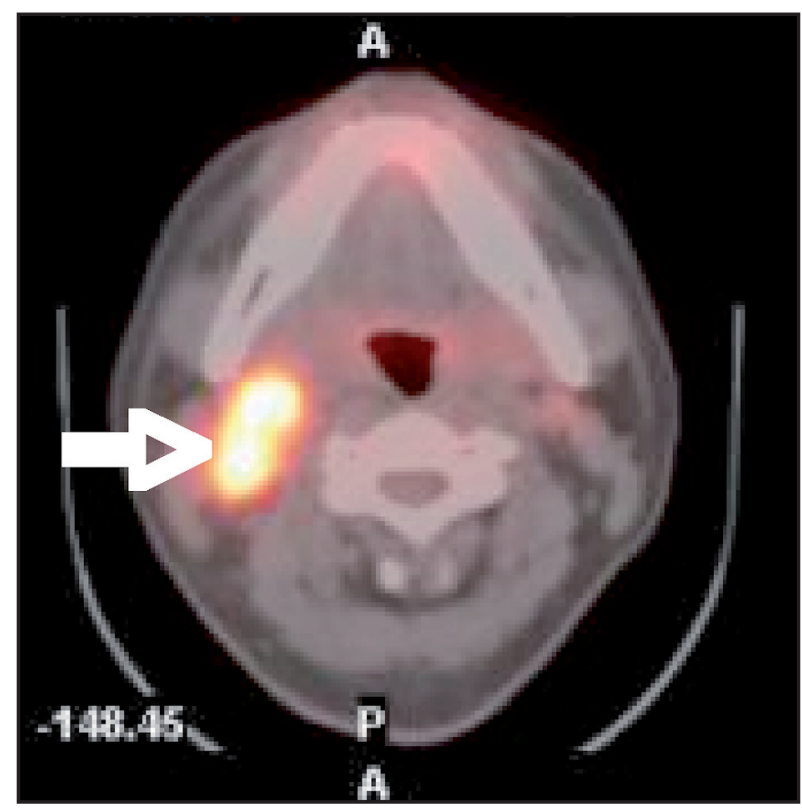

Figura 2. Imagen PET en donde se advierte la presencia de adenopatías laterocervicales derechas hipermetabólicas (flecha blanca).

Se practicó exéresis total de las adenopatías laterocervicales. El diagnóstico anátomo-patológico fue de linfadenitis granulomatosa necrotizante. No se observaron BAAR, ni hongos ni signos de vasculitis. Se inició tratamiento con antiinflamatorios no esteroideos y posteriormente con corticoides a dosis de $0,5 \mathrm{mg} / \mathrm{kg} /$ día logrando mejoría franca sin llegar a desaparecer las adenopatías. Tres meses después de la intervención presentó supuración por un punto de la herida quirúrgica, con frotis que mostró crecimiento de estafilococo coagulasa negativo, iniciándose tratamiento con amoxicilinaácido clavulánico $500 \mathrm{mg}$ cada 8 horas. Se realiza nuevo TC de cuello que puso de relieve el trayecto fistuloso laterocervical derecho, sin identificarse colecciones cervicales profundas, siendo visible el conglomerado adenopático entre los niveles IIA, IIB y III unilaterales derechos. El examen radiológico puso de manifiesto que las adenopatías continuaban presentando pequeñas zonas hipodensas múltiples de aspecto necrótico. Teniendo en cuenta la procedencia del paciente, la localización unilateral y el aspecto de los ganglios patológicos se sugirió el diagnóstico de EK. No obstante se solicitó PPD que resultó ser positivo y se realizaron estudios de quantiferon y PCR para TBC a partir de la biopsia de las adenopatías. Fueron positivos realizándose, por consiguiente, el diagnóstico de tuberculosis ganglionar. Se inició tratamiento tuberculostático con buena evolución clínica.

\section{DISCUSIÓN}

La linfadenitis cervical tuberculosa es la manifestación más común de TBC en cabeza y cuello y representa aproximadamente el $15 \%$ de los casos de TBC extrapulmonar. Debido al síndrome de inmunodeficiencia adquirida, al abuso de drogas intravenosas y al incremento de la inmigración, la prevalencia de tuberculosis se ha incrementado en países industrializados ${ }^{1-2}$. Afecta principalmente a niños y adultos jóvenes y presenta una discreta predilección por el sexo femenino $0^{2-3}$.

Por otra parte la enfermedad de Kikuchi es una enfermedad rara que consiste en una linfadenitis necrotizante histocitiaria. Aunque las primeras descripciones fueron en personas de origen asiático, como el caso que nos ocupa, la enfermedad ha sido descrita en individuos de todas las razas. Al igual que la linfadenitis cervical tuberculosa, predomina discretamente en mujeres ${ }^{4}$ y la mayoría 


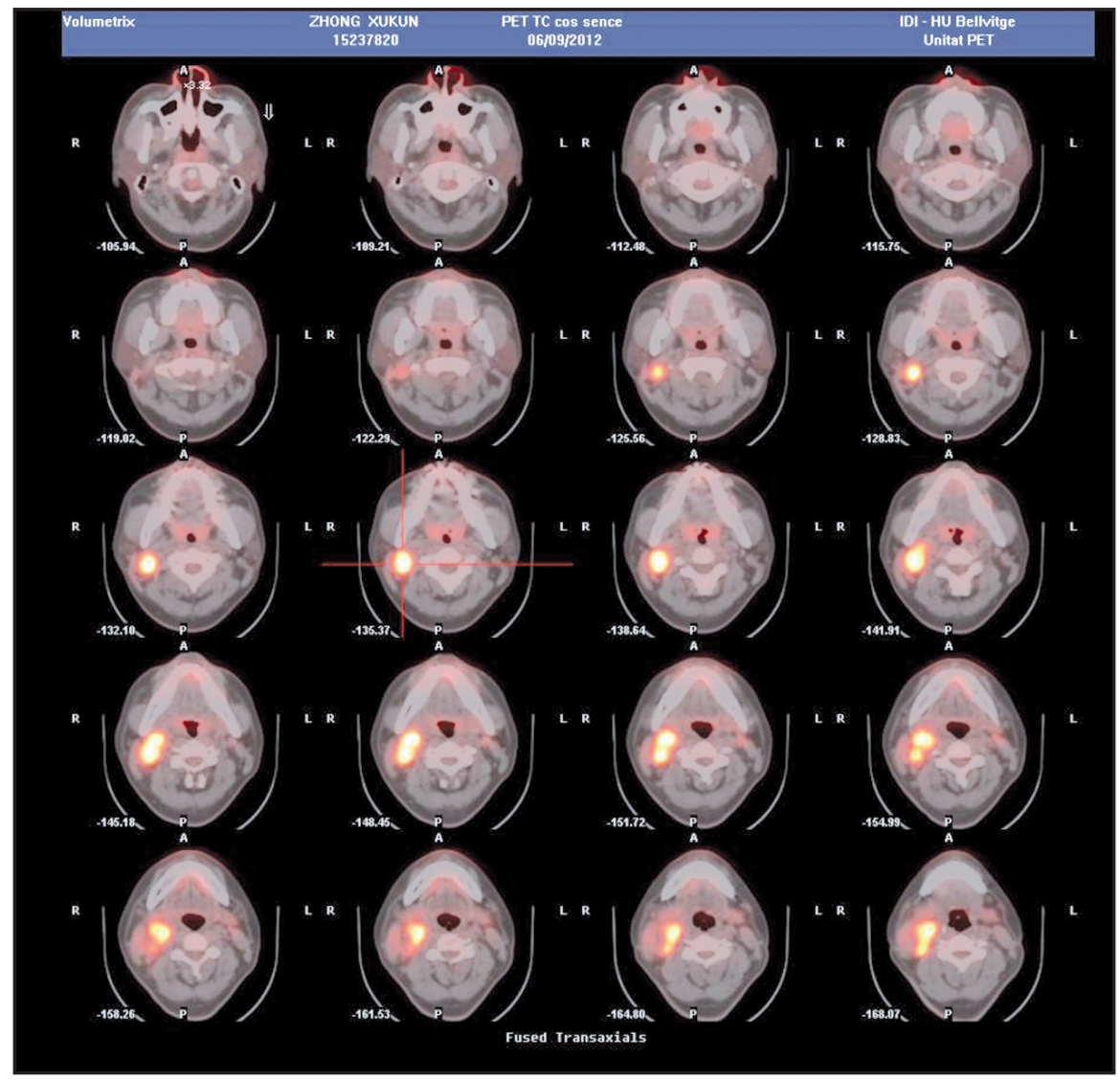

Figura 3.

de los pacientes son jóvenes menores de 30 años.

La presentación clínica en ambas entidades suele consistir en una o más masas cervicales dolorosas, que pueden ser duras o fluctuantes, asociadas a fiebre y sudoración nocturna ${ }^{5}$. Usualmente presentan pocos síntomas constitucionales al examen físico. En el caso de la EK el proceso suele ser autolimitado.

Los hallazgos por imagen no permiten distinguir de manera absoluta la linfadenitis tuberculosa de la EK. Es importante para interpretar los hallazgos conocer el origen demográfico del paciente y su estado inmunológico.

Los hallazgos de TC en la linfadenitis tuberculosa cervical son variables y dependen del grado de caseificación presente en el nódulo adenopático. Los nódulos linfáticos pueden aparecer inicialmente aumentados de tamaño, con atenuación similar al músculo y captación homogénea de contraste.
Durante el curso de la enfermedad, una vez se desarrolla la caseificación, los nódulos muestran una baja densidad central por necrosis, con realce periférico y eventualmente se vuelven quísticos. Además usualmente se asocian a discretos cambios inflamatorios adyacentes ${ }^{6}$. En pacientes tratados por tuberculosis se suelen ver adenopatías calcificadas. El proceso es comúnmente bilateral y afecta con mayor frecuencia al triángulo posterior y a los ganglios de la cadena yugular interna ${ }^{7}$. La RM puede ser útil en la evaluación de la actividad y respuesta al tratamiento, sin embargo es una técnica limitada para detectar calcificaciones, lo cual es un importante criterio diagnóstico para linfadenitis tuberculosa, que ayuda a diferenciarla de otras enfermedades benignas y malignas ${ }^{7}$. No se han descritos casos de LT tuberculosa con PET.

Entre los hallazgos de TC descritos en un estudio de 96 pacientes con EK$^{8}$ se incluían 
múltiples nódulos linfáticos unilaterales, aumentados moderadamente de tamaño, homogéneos y con infiltración perinodal con afectación principalmente de los niveles II-V. También se ha descrito la necrosis adenopática. Cuando el TC revela necrosis nodal, la EK puede simular otras enfermedades como metástasis y tuberculosis. Mediante el seguimiento con TC de estos pacientes se observó una regresión parcial o completa de la linfadenopatía en algunos meses, lo que demuestra su carácter autolimitado, que lo diferencia de la linfadenitis tuberculosa cervical y de otras patologías malignas como el linfoma y las metástasis ${ }^{8}$.

En un estudio realizado en 24 pacientes con EK y 45 pacientes con LT, se valoraron las diferencias del patrón de necrosis nodal observado entre estas dos entidades en el TC para facilitar su diagnóstico. La extensión de necrosis nodal fue moderada en el $87,5 \%$ de los 24 pacientes con EK y severa en el $62,2 \%$ de los 45 pacientes con LT. La extensión de la necrosis nodal fue significativamente diferente entre EK y LT, ya que el $91,6 \%$ de pacientes con EK tenían focos necróticos múltiples en nódulos aumentados de tamaño, mientras que el $44,4 \%$ de pacientes con LT tenían un foco necrótico único dentro de una adenopatía. No hubo diferencias significativas en la localización del foco necrótico dentro del nódulo más grande entre las dos enfermedades. En ambas enfermedades, el foco necrótico fue frecuentemente localizado en la porción periférica dentro del nódulo. Los márgenes del foco necrótico fueron indistintivos en el 79,2\% de Ios pacientes con EK y relativamente bien definidos en el $80 \%$ de los pacientes con LT. Diferencias significativas entre las dos enfermedades fueron observadas en los márgenes del foco necrótico. No hubo diferencia significativa en la infiltración perinodal entre EK y LT. Las calcificaciones dentro de los nódulos fue observada en 11 de 45 pacientes con $\mathrm{LT}^{9}$.

A pesar de los hallazgos imagenológicos, el diagnóstico preciso de enfermedad de Kikuchi sobre frotis citológico es crucial, ya que éste puede ser confundido con linfadenitis tuberculosa, lupus eritematoso sistémico, linfoma maligno o incluso carcinoma metastásico, evitando investigaciones innecesarias y tratamientos potencialmente perjudiciales ${ }^{10}$.
Igualmente para el diagnóstico de LT es importante llevar a cabo una biopsia del nódulo linfático afecto y examinarlo histológica y/o microbiológicamente. Un test de tuberculina en piel debe ser realizado. En un estudio realizado con 23 pacientes con LT, todos ellos, a excepción de dos, tuvieron un test de piel fuertemente positivo a la tuberculina ${ }^{3}$. En la realización de una biopsia de los ganglios linfáticos cervicales en pacientes sospechosos se debe combinar además estudios histopatológicos, PCR para TBC y cultivo de BAAR simultáneamente ${ }^{11}$.

\section{CONCLUSIÓN}

La enfermedad de Kikuchi es una entidad que usualmente se manifiesta con adenopatías laterocervicales con pequeños focos de necrosis de manera unilateral. Si bien estas características son propias de esta patología, hay que considerar siempre la posibilidad de la tuberculosis ganglionar, en primer lugar porque es una entidad más frecuente, y en segundo lugar porque la propia tuberculosis puede simular una afectación ganglionar de similar aspecto. Por consiguiente, ante la presencia de adenopatías que induzcan a pensar en el diagnóstico de linfadenitis necrotizante histiocitaria, es necesario descartar la tuberculosis como primera opción diagnóstica.

\section{BIBLIOGRAFÍA}

1. Millek WT. Tuberculosis in the 1990. Radiol Clin North 1994; 32: 649-61.

2. Jha BC, Dass A, Nagarkar NM et-al. Cervical tuberculous lymphadenopathy: changing clinical pattern and concepts in management. Postgrad Med J 2001; 77 (905): 185-7.

3. Ueda T, Murayama T, Hasegawa $Y$, Bando K. Tuberculous lymphadenitis: a clinical study of 23 cases. Kekkaku 2004; 79(5): 349-54.

4. MJ Bennie, KM Bowles, SC Rankin. Necrotizing cervical lymphadenopathy caused by KikuchiFujimoto disease. The British Journal of Radiology 2003; 76: 656-8.

5. LEE JK. Computed body tomography with MRI correlation. Lippincott Williams \& Wilkins. $4^{\text {th }}$ ed. Volume 1. 2006; 193-9. 
6. Som PM, Brandwein MS. Lymph nodes. In: Som PM, Curtin HD, eds. Head and Neck Imaging. $4^{\text {th }}$ ed. St Louis: Mosby-Year Book 2003; 1865-934.

7. Woo KM, Moon HH, KeE HC. CT and MR Imaging of head and neck tuberculosis. Radiographics 1887; 13: 391-402.

8. Soon- Young Kwon, TaIK-Kun KIM, Young-SIK KIM, KI Yeol Lee, Nam Joon Lee, and Hae Young Seol. CT findings in Kikuchi disease: Analysis of 96 cases. AJNR 2004; 25: 1099-102.

9. Lee S, Yoo JH, Lee SW. Kikuchi Disease: Differentiation from Tuberculous Lymphadenitis
Based on Patterns of Nodal Necrosis on CT. AJNR Am J Neuroradiol 2012; 33(1): 13540.

10. Sivakumar S, Ramamoorthy R. Fine needle aspiration cytology of Kikuchi-Fujimoto's disease: a report of 2 cases with emphasis on cytologic features and differential diagnosis. Acta Cytol2012; 56(4): 457-62.

11. Mada Y, Uek Y, Konno A. A clinical study on 10 cases of cervical tuberculous lymphadenitis. Nihon Jibiinkoka Gakkai Kaiho 2012; 115(11): 950-6. 